\title{
Spin and charge stripes in trilayer nickelates
}

Daniel Phelan ${ }^{a}$, J. Zhanga,b, Y.S. Chen ${ }^{c}$, D.M. Pajerowski ${ }^{\text {, }}$, H. Zheng ${ }^{a}$, A.S. Botana ${ }^{a}$, L. Harrigere, J. Rodriguez-Rivera $^{\text {e }}$, J.P.C. Ruff ${ }^{\text {, N.J. Schreiber }}{ }^{g}$, B. Wang ${ }^{a}$, M.R. Normana, S. Rosenkranz ${ }^{a}$, J. F. Mitchell ${ }^{a}$

${ }^{a}$ Materials Science Division, Argonne National Laboratory, dphelan@anl.gov

bMaterials Science and Technology Division, Oak Ridge National Laboratory

${ }^{c}$ ChemMatCARS, The University of Chicago

${ }^{\mathrm{d}}$ Quantum Condensed Matter Division, Oak Ridge National Laboratory

eNIST Center for Neutron Research, National Institute of Standards and Technology

${ }^{\mathrm{f}} \mathrm{CHESS}$, Cornell University

9Department of Materials Science and Engineering, Cornell University

Highly orbitally polarized trilayer nickelates represent a potential platform for searching for high$T_{C}$ superconductivity [1]. $\mathrm{La}_{4} \mathrm{Ni}_{3} \mathrm{O}_{8}$, which has square-planar coordinated nickel, is one such material. It undergoes a semiconductor-insulator transition on cooling at $T_{S I} \sim 105 \mathrm{~K}$, which is accompanied by formation of charge stripes evidenced by X-ray scattering measurements [2]. These stripes will be discussed along with our recent finding that the ground state is magnetic and consists of antiferromagnetic spin stripes that are commensurate with the charge stripes [3]. The magnetic stripes are modeled as occurring within uncorrelated trilayers. Comparison of the charge and spin stripe order parameters reveals that, in contrast to single-layer nickelates and most transition metal oxides, the orders both appear at $T_{S I}$, suggesting a strong coupling between the two.

*This work was supported by the U.S. Department of Energy, Office of Science, Basic Energy Sciences, Materials Science and Engineering Division.

\section{$\underline{\text { References }}$}

[1] J. Zhang, A. S. Botana, J. W. Freeland, D. Phelan, H. Zheng, V. Pardo, M. R. Norman, and J. F. Mitchell, Nat. Phys. 13, 864 (2017).

[2] J. Zhang, Y. S. Chen, D. Phelan, H. Zheng, M. R. Norman, and J. F. Mitchell, Proc. Natl. Acad. Sci. U. S. A. $113,8945(2016)$.

[3] J. Zhang, D. M. Pajerowski, A. S. Botana, L. Harriger, J. Rodriguez-Rivera, J. P. C. Ruff, N. J. Schreiber, B. Wang, Yu-Sheng Chen, M. R. Norman, S. Rosenkranz, J. F. Mitchell, and D. Phelan, arXiv:1903.03246 (2019). 